\title{
The kinetics of formation and morphological memory of polymeric nanostructures
}

\author{
I. V. Melikhov ${ }^{1}$, O. V. Alekseeva ${ }^{2}$, V. N. Rudin ${ }^{1}$, E. D. Kozlovskaya ${ }^{1}$, A. V. Noskov² \\ ${ }^{1}$ M. V. Lomonosov Moscow State University, Moscow, Russia \\ ${ }^{2}$ G. A. Krestov Institute of Solution Chemistry of RAS, Ivanovo, Russia \\ melikhov@radio.chem.msu.ru, ova@isc-ras.ru
}

\section{PACS 81.05.Lg}

DOI 10.17586/2220-8054-2015-6-4-551-561

\begin{abstract}
The mesokinetic model of the polymeric body formation was formulated, describing the nucleation, growth and aggregation of macromolecules followed by the aggregates' assembly into the nanostructured body. According to this model, the near-surface nanostructure of the polymeric body retains the information on how it was formed, and this information can be extracted from the morphological characteristics of the microrelief of the body while maintaining its integrity, that is, body has the morphological memory. The mesokinetic model was used to study film formation by the evaporation of an $o$-xylene or toluene solution of polystyrene, which provided an opportunity to identify the kinetics of nanostructure formation found in the surface area of the film after completion of the process. It turned out that at the beginning of evaporation, a number of the primary macromolecules in the form of spheroidal nanoparticles were formed, and then, the primary aggregates coalesced into the secondary aggregates forming the ordered chains of nanoparticles.
\end{abstract}

Keywords: polystyrene, microrelief, primary (nanoparticles) and secondary (microparticle) units.

Received: 9 April 2015

\section{Introduction}

According to the electron micrographs of polymers (films, granules, membranes, etc.) published in the literature, many of these substances have nanostructured microrelief. We observed such microrelief on the surface of polystyrene granules purchased from "Aldrich" (Germany), as indicated by Fig. 1, which shows that the near-surface areas of the studied granules have a multilevel hierarchical nanostructure. They consist of aggregates of polystyrene molecules in the form of spheroidal nanoparticles, aggregated into the chains, combined into microparticles, which form a granule. Fig. 1 leads to the question, whether the microrelief of the polymeric body retains information about how it was formed. Is it possible, focusing on electron microscopic images of the microrelief, to determine the speed of the elementary processes that lead to this microrelief? As it is known, the exact answer to this question may be provided by studying the kinetics of elementary processes for monomer integration into oligomer and polymer molecules, the formation of aggregates from polymer molecules (associates, floccules, colloidal particles) and aggregates association in a polymeric body (solidification, crystallization) [1-8]. Methods of studying of these elementary processes have been developed and are widely used [3-11]. In this case, the design of experiments and interpretation of the results is based on kinetic models that enable the successful description of many experimental results [11-15], but do not allow their quantitative prediction, as each model inevitably contains arbitrary assumptions that reduce its predictive ability. With this in mind, this article attempts to formulate a mesokinetic model in which the only arbitrary assumption is the crucial role of binary collisions of particles in the system, and other assumptions are included in the unknown frequency functions and are available for 
experimental determination. The proposed model was used to study the microrelief of the films formed by evaporation of a polystyrene solution (Fig. 2), which led to the concept of morphological film memory. The study of the microrelief of granules from Aldrich (Fig. 1) as well as the study of the electron-microscopic images of many polymeric bodies described in the literature has led to the same conclusion. This enabled us to assert that many polymeric bodies possess morphological memory which is different from the shape memory and other types of polymer memory [16-18] with the memory carrier being localized at the body surface, rather than within its volume.

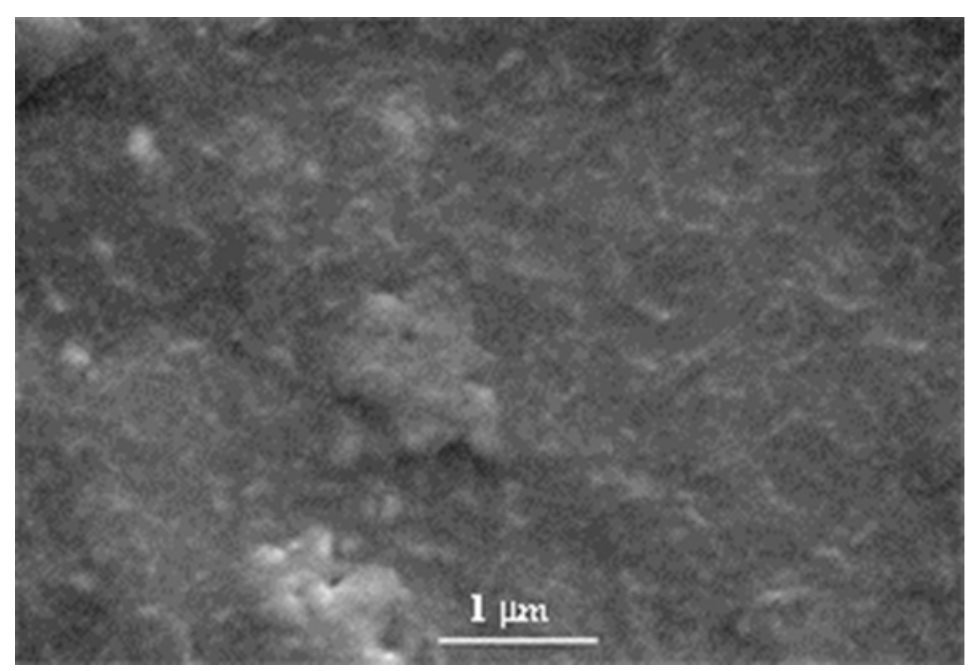

FiG. 1. Electron micrograph of the surface area of a typical polystyrene granules from "Aldrich" (Germany). Microscope CamScan, a sample was prepared by the standard method

\section{Model of nanostructure formation}

The processes in a homogeneous solution of a monomer A which evaporates at a predetermined speed are studied. At the time $t=0$ the polymerization initiator $\mathrm{B}$ is added into the solution, which leads to the formation of polymeric molecules which combine together to form ordered aggregates. Upon evaporation of the solution, its volume decreases, leading to an increase in the concentration of molecules and aggregates, which do not evaporate. During the evaporation, an increasing number of the molecules and aggregates is brought into contact with each other, and at the moment $t_{F}$ of the complete removal of the solvent they form a polymer body with the following hierarchical structure, its nanolevel consisting of polymer molecules, microlevel - by the aggregates, and macrolevel - by the body itself.

At $0<t<t_{F}$ the solution contains monomer molecules $(j=0)$, polymer molecules $(j=1)$ and aggregates $(j=2,3 \ldots)$.

The state of each particle is characterized by parameters $\vec{X}=\left\{n_{1} \ldots n_{i} \ldots n_{m}, v\right.$, $\left.X_{1} \ldots X_{i} \ldots X_{p}\right\}$, where $n_{i}$ is the number of A molecules, which have formed one of the $\mathrm{m}$ of polymer molecules of the particle, with a total number of the A molecules equal to $n=\sum_{i-1}^{m} n_{i} ; v$ is a particle volume; $X_{i}$ is a parameter of shape, composition or structure of the particle. The condition for the conservation of the number of atoms introduced into the 


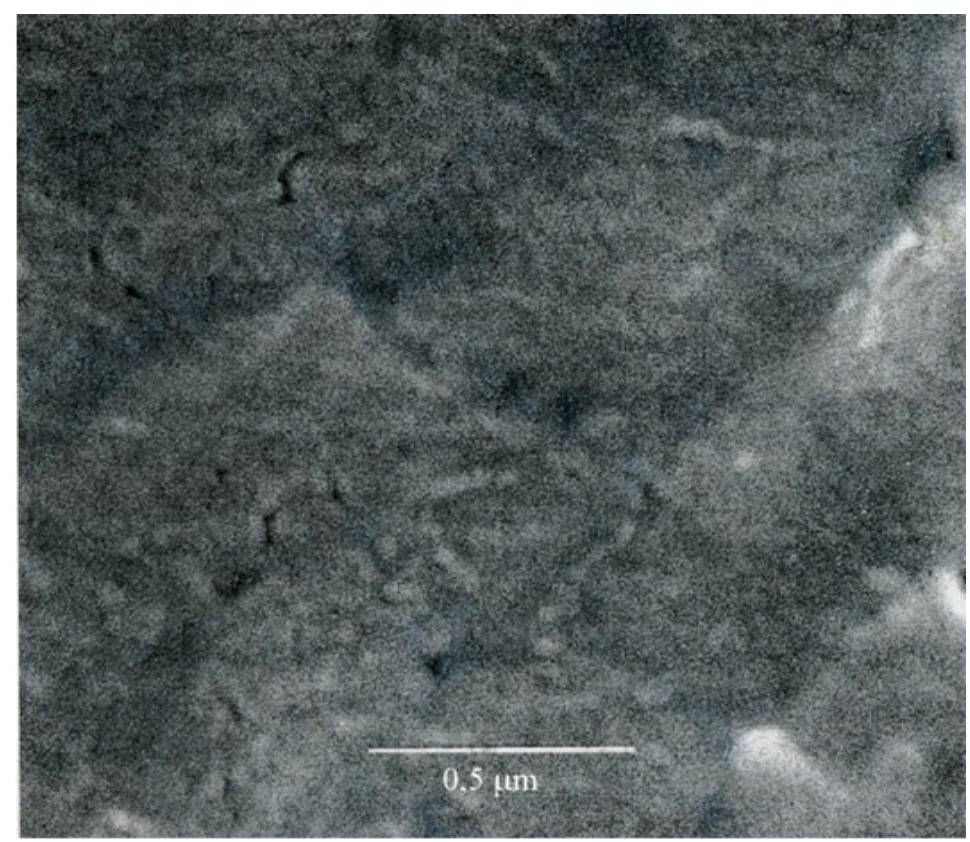

FIG. 2. Microrelief of the film obtained by removing the solvent from the $o$-xylene solution of polystyrene. The conditions of film formation: $V_{0}=36,5 \mathrm{~cm}^{3}, T=293 \mathrm{~K}$, initial concentration of polystyrene in the solution $M_{0}=0.15 \mathrm{~g} / \mathrm{cm}^{3}, S=1200 \mathrm{~cm}^{2}$. Microscope CamScan

system in the molecules $\mathrm{A}$ is fulfilled:

$$
C_{A 0} V_{0}=\int_{V}\left[C_{A}(1-\varepsilon)+\sum_{j} \sum_{n} n N_{j n}(t)\right] d V,
$$

where $C_{A 0}$ and $C_{A}$ are the initial and the current concentration of the A molecules in the solution; $V_{0}$ and $V$ are the initial and the current volume of the system; $\varepsilon$ is the share of the system volume occupied by the polymer molecules and aggregates; $N_{j n}(t)$ is the concentration of macromolecules and aggregates, including $n$ molecules of $\mathrm{A}$ by the time $\mathrm{t}$.

The volume $V$ is equal to:

$$
V=V_{0} \int_{0}^{t} v_{L} S \omega_{S} d t
$$

where $v_{L}$ is the volume change after the evaporation of one of the solvent molecules; $S$ is the area of the evaporation surface; $\omega_{S}$ is the flow of solvent molecules in the vapor at the moment $t$.

At $0<t<t_{F}$ the concentration $N_{j n}(t)$ in each system area changes due to the associations of the particles, because of the binary collisions and fragmentation of molecules and aggregates under the influence of thermal motion and mechanical effects. Therefore:

$$
\Delta N_{j n}(t)=\left(\Omega_{j+}-\Omega_{j-}-\operatorname{div} \vec{J}_{j n}\right) \Delta t
$$

where $\Delta N_{j n}(t)$ is the change of the $N_{j n}(t)$ between the time moments of $t$ and $t+\Delta t ; \Omega_{j+}$ and $\Omega_{j-}$ are the frequency functions that characterize the processes that lead to an increase and decrease of $n$ of the particles of $j^{t h}$ type; $\vec{J}_{j n}$ is the flow of the particles in the condition $(j, n)$ through the given area. 
Therein:

$$
\begin{gathered}
\Omega_{j+}=\alpha_{j n-1} N_{j n-1} C_{A}+\alpha_{j-1, n-a} N_{j-1, n-a} N_{j-1, a}+\sum_{k \geqslant j} \beta_{k, n+b} N_{k, n+b}, \\
\Omega_{j-}=\alpha_{j n} N_{j n} C_{A}+\sum_{k>j} \alpha_{j n} N_{j n} N_{k, a}+\beta_{j n} N_{j n},
\end{gathered}
$$

where $N_{j n}=N_{j n}(t) ; \alpha_{j n}$ and $\beta_{j n}$ are functions that characterize the frequency of association and fragmentation of particles; $a$ and $b$ are the characteristic changes of $n$ during a single act of unification and fragmentation.

With the known frequency functions $\alpha_{j n}(n, t)$ and $\beta_{j n}(n, t)$, equations (1)-(5) allow one to calculate the volume fraction of the area filled with the particles of $j^{\text {th }}$ type:

$$
\varepsilon_{j}(t)=\sum_{n} v_{A j} n N_{j n}(t)
$$

where $v_{A j}$ is the volume per single A molecule after its transition into the polymer molecule of the $j^{\text {th }}$ type particle.

From relations (4)-(6), it follows that moving from the concentration $N_{j n}(t)$ to the distribution function $\varphi_{j n}=\partial N_{j n} / \partial n$ and expanding $\alpha_{j n} \varphi_{j n}$ and $\beta_{j n} \varphi_{j n}$ in Taylor series limiting to three terms of the expansion, equation (3) can be written as:

$$
\frac{\partial \varphi_{j n}}{\partial t}=\frac{\partial^{2}\left(D_{j n} \varphi_{j n}\right)}{\partial n^{2}}-\frac{\partial\left(G_{j n} \varphi_{j n}\right)}{\partial n}+W_{j k}-\operatorname{div} \vec{J}_{j n}
$$

where $D_{j n}=\frac{1}{2}\left(a^{2} \alpha_{j n} C_{A}+b^{2} \beta_{j n}\right) ; G_{j n}=a \alpha_{j n} C_{A}-b \beta_{j n}$. $W_{j k}$ is the function of the transition of the particles of $j^{\text {th }}$ type in the groups of particles of other $k^{t h}$ types, related by the frequency functions in accordance with the conditions (4) and (5).

Therein:

$$
\varepsilon_{j}(t)=\int_{n>n_{j 0}} v_{A j} n \varphi_{j n}(t) d n
$$

where $n_{j 0}$ is a number of molecules A, forming a particle of minimum size, to which the speed of enlargement $G_{j n}$ can be attributed.

The solution of equation (7) with the corresponding boundary conditions can provide the ability to search the particle distribution function for a status parameter $\vec{X}$, which can be expressed as $\varphi_{j}(\vec{X}, t)=\varphi_{j n}(t) f_{j}(v)_{n} f_{j}\left(X_{1}\right)_{n} \ldots f_{j}\left(X_{i}\right)_{n v} \ldots f_{j}\left(X_{p}\right)_{n v}$, where $f_{j}(v)_{n}$ and $f_{j}\left(X_{i}\right)_{n v}$ are the distribution density on $v$ of the particles with the given $n$ and the distribution density on $X_{i}$ of the particles with the given $n$ and $v$.

\section{Morphological body memory model}

Within the framework of the considered model, the microrelief of the body at time $t$ is represented by a set of ordered aggregates, among which the molecules are located, with the degree of filling of the volume of the body's surface area by particles of:

$$
\varepsilon_{S}=\int_{n>n_{j 0}} v_{A j} n \varphi_{j n}\left(t_{F}\right)_{S} d n
$$

where $\varphi_{j n}\left(t_{F}\right)_{S}$ is the distribution on $\mathrm{n}$ of particles of the $j^{\text {th }}$ type on the body's surface area at the moment $t_{F}$. Distribution $\varphi_{j n}\left(t_{F}\right)_{S}$, as well as any distribution on any area of the system, is an integral of equation (7) and is determined by which of the frequency functions $\alpha_{j n}\left(n, t_{F}\right)$ and $\beta_{j n}\left(n, t_{F}\right)$ occurred at $t \rightarrow t_{F}$. Consequently, the information about the functions $\alpha_{j n}\left(n, t_{F}\right)$ and $\beta_{j n}\left(n, t_{F}\right)$, included in this integral, can be extracted from the 
distribution $\varphi_{j n}\left(t_{F}\right)_{S}$. Taking this into account, functions $\varphi_{j n}\left(t_{F}\right)_{S}$ can be considered as carriers of the body morphological memory of the process which during the time $t_{F}$ has led its microrelief into the state represented by the equation (9). According to equations (1)-(8), the frequency functions depend on the system properties $\vec{y}=\left\{C_{A 0}, V_{0}, S, \omega_{S}\right\}$, accordingly the microrelief saves the information about the conditions in which the body was formed.

The distribution $\varphi_{j n}\left(t_{F}\right)_{S}$ can be variable. For the formation of macromolecules not prone to aggregation in a homogeneous system under the condition of independence of $\alpha_{j n}$ and $\beta_{j n}$, this distribution on $\mathrm{n}$ is determined by integrating the equation ( 7 ) in the form:

$$
\frac{\partial \varphi_{j n}}{\partial t}=D_{1 n} \frac{\partial^{2} \varphi_{1 n}}{\partial n^{2}}-G_{1 n} \frac{\partial \varphi_{1 n}}{\partial n} .
$$
formula

If the conditions $\vec{y}$ are such that $\alpha_{1 n} C_{A}>>\beta_{1 n}$, integration leads $[19,20]$ to the

$$
\varphi_{1 n}\left(t_{F}\right)_{S}=C_{B 0}\left[(\pi A p)^{-1 / 2} \exp \left(-X_{-}^{2}\right)-\frac{1}{2 p} \exp \left(\frac{n-n_{10}}{p}\right) \operatorname{erfc}\left(X_{+}\right)\right],
$$

where $C_{B 0}$ is the initiator concentration in the initial solution:

$$
A=\int_{0}^{t} G_{1 n} d t ; p=D_{1 n} / G_{1 n} ; X_{ \pm}=\left(n-n_{0 j} \pm A\right) /(4 \pi A p)^{1 / 2} ; \operatorname{erfc}\left(X_{+}\right)=\frac{2}{\sqrt{\pi}} \int_{X_{+}}^{\infty} e^{-X^{2}} d X
$$

Equation (11) describes the molecular weight distribution of the polymer molecules forming the microrelief of the body, provided that the body was formed according to the model (1)-(8) in the independence of $D_{1 n}$ and $G_{1 n}$ on $n$. Given this, we can assume that in systems where the experimental data on the microrelief is described by relation (11), this condition is realized, and the information about it is accumulated by the distribution $\varphi_{1 n}\left(t_{F}\right)_{S}$.

When $t>t_{F}$, the distribution $\varphi_{1 n}\left(t_{F}\right)_{S}$ can change under the influence of various factors, so that going from $n$ to $\vec{X}$ :

$$
\varphi_{j}(\vec{X}, \tau)_{S}=\varphi_{j n}\left(t_{F}\right)_{S} P_{j}\left(\vec{X}, t_{F}, \tau\right)
$$

where $\varphi_{j}(\vec{X}, \tau)_{S}$ is the distribution function on $\vec{X}$ of the near-surface particles of the $j^{t h}$ type after influences on the body over time $\tau ; P_{j}\left(n, t_{F}, \tau\right)$ is the indicator of the microrelief stability to external influences.

Function $P_{j}\left(\vec{X}, t_{F}, \tau\right)$, which characterizes the degree of preservation of the body's morphological memory after its use, can be defined in the framework of our model after detailing of the respective frequency functions.

From the above, it follows that if after the formation and use of the body the microrelief remains on its surface, allowing one to experimentally determine the distribution functions of the structural elements of the microrelief on morphological parameters of the state and to present them as a solution of the equation (7), it can be argued that the body has morphological memory. According to the electron micrographs published in the literature, there are several polymeric bodies exhibiting morphological memory. Such a memory is also possessed by many inorganic nanostructured materials, although their nanoparticles, after aggregation, often become indistinguishable. 


\section{Experimental study of polystyrene film formation. Methods and results}

In conducting this research, we used a previously-described technique [21,22]. The polystyrene granules from Aldrich were dissolved in a solvent capable of rapid evaporation under normal conditions. A portion of the solution with a predetermined polystyrene content was introduced into a flat glass cuvette, which was then placed in the thermostatic chamber of a scale for continuous weighing, so that the weight change of the cuvette with the solution during the evaporation of the solvent was observed. Evaporation was carried out so that only the molecules of the solvent evaporated, making it possible to determine the rate at which the concentration of the polystyrene solution increased by measuring the rate at which the mass of the cuvette decreased. When the mass of the cuvette stopped changing, the formed polymer film was removed from the cuvette, its near-surface areas were studied using a scanning electron microscope, and the distribution functions of particle images of its microrelief on size were determined: $\theta_{k}(\ell)=B_{k}(\ell) / B_{k 0}$, where $B_{k}(\ell)$ is the number of measured particles of $k^{\text {th }}$ type, that are smaller than $\ell=s^{1 / 2}$ at the area of the image of $s ; B_{k 0}$ is the total number of particles measured. The particles which had homogeneous images were taken for primary nanoparticles $(\mathrm{k}=1)$, the ordered clusters of primary particles were taken for the aggregates (microparticles, $\mathrm{k}=2$ ), and the groups of orderly contacted assemblies were taken for the secondary aggregates $(\mathrm{k}=3)$. In some experiments, a solution of $\mathrm{C}_{60}$ was added to the initial solution of polystyrene, and then the action was taken as described above.

The experiments have shown that the rate of the solvent removal from the cuvette was varied in two steps, as can be seen in Fig. 3, which shows the data on weight changes of the toluene solution of polystyrene in the cuvette. Therein the condition was fulfilled:

$$
J=J_{0} \Delta+J_{1} \exp \left[-\omega\left(t-t_{1}\right)\right](1-\Delta),
$$

where $J_{0}$ and $J_{1}$ are flows of evaporated solvent molecules from the surface of the cuvette at $t<t_{1}$ and $t=t_{1} ; \Delta=1$ at $t<t_{1}$ and $\Delta=0$ at $t>t_{1} ; J_{0}=2,06 \cdot 10^{-3} \mathrm{~mol} /\left(\mathrm{m}^{2} \cdot \mathrm{s}\right)$; $J_{1}=2,53 \cdot 10^{-3} \mathrm{~mol} /\left(\mathrm{m}^{2} \cdot \mathrm{s}\right) ; \omega=(2,3 \pm 0,5) \cdot 10^{-3} \mathrm{c}^{-1}$ is the empirical parameter.

By the end of the second step, when the evaporation was almost complete, a polymer film was formed in the cuvette which had properties that did not alter after transferring it to an electron microscope. Microrelief of the film was created by the microparticles, single or combined into the chains spheroidal nanoparticles were found on their surface and between them (Fig. 2). For the microparticles and their constituent nanoparticles the functions $\theta_{k}(l)$ are presented as functions of $\mathrm{Z}$, shown in Fig. 4 , where $Z=\left(l-l_{k 0}\right) /\left(l_{k M}-l_{k 0}\right), l_{k 0}$ is the minimum size of measured particles of $k^{\text {th }}$ type; $l_{k M}$ is the size corresponding to the condition $\theta_{k}\left(l_{k M}\right)=0,5$ (Table 1$)$. The corresponding differential distribution functions $\psi_{k}(l)=\frac{\partial \theta_{k}(l)}{\partial l}$ are described by the formula:

$$
\psi_{k}(l)=\left[\left(\pi A_{k} p_{k}\right)^{-1 / 2} \exp \left(-X_{-}^{2}\right)-\frac{1}{2 p_{k}} \exp \left(\frac{l-l_{k 0}}{p_{k}}\right) \operatorname{erfc}\left(X_{+}\right)\right],
$$

where $A_{k}, p_{k} \quad l_{k 0}$ are empirical parameters, some of which are listed in the Table $2 ; X_{ \pm}=$ $\left(l-l_{k 0} \pm A_{k}\right) /\left(4 \pi A_{k} p_{k}\right)^{1 / 2}$.

Functions $\theta_{1}(l)$ of nanoparticles, included in the microparticles of different sizes, were similar. They changed when fullerene was introduced into the system (Fig. 4). In particular, during the formation of the film using an $o$-xylene solution of polystyrene at $V_{0}=36,5 \mathrm{~cm}^{3}$, $M_{0}=0,15 \mathrm{~g} / \mathrm{cm}^{3}$ and initial fullerene concentration of $M_{C}=10^{-5}-10^{-4} \mathrm{~g} / \mathrm{cm}^{3}$, the function $\theta_{1}(l)$ shifted toward larger units provided the following:

$$
l_{1 M}=l_{M 0}\left(1+5,1 \cdot 10^{2} M_{C} / M_{0}\right),
$$




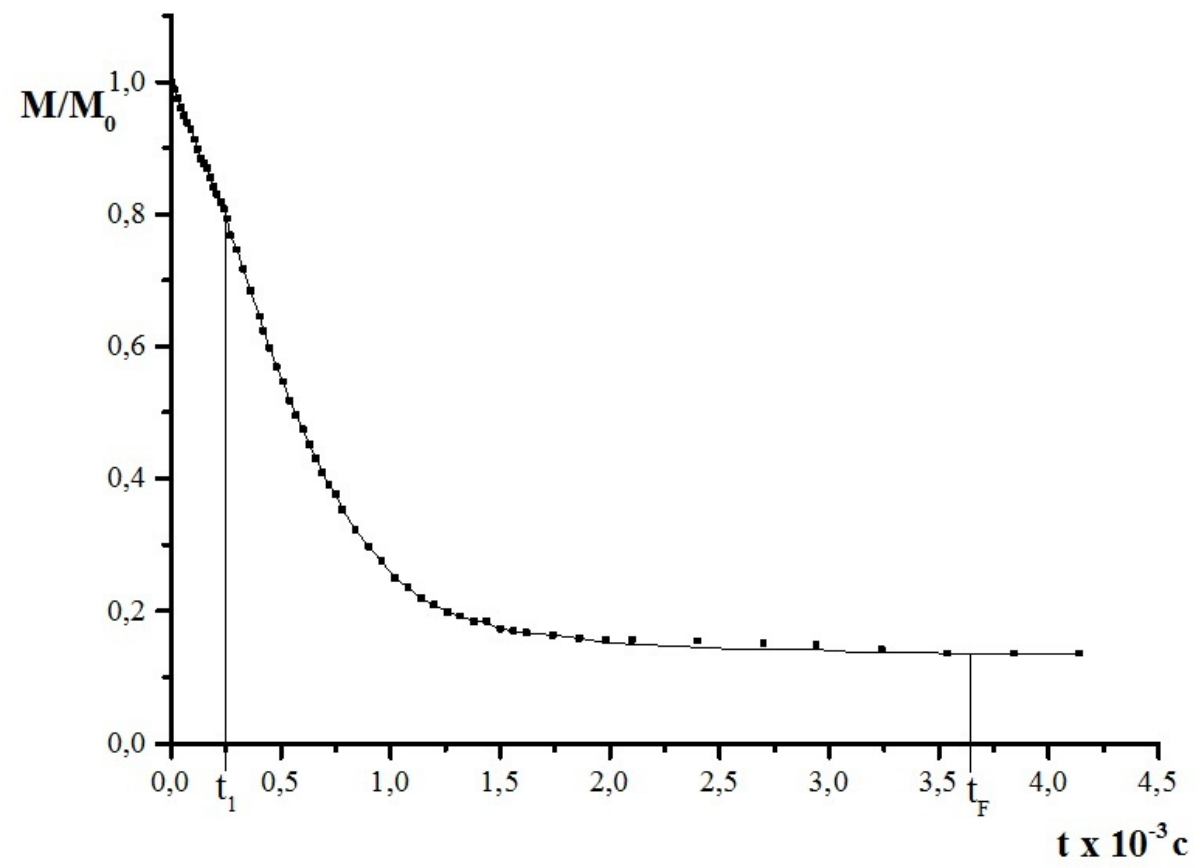

FIG. 3. Change of mass $M$ of the polystyrene solution in toluene. The experimental conditions: solvent: toluene, $T=293 \mathrm{~K}, V_{0}=0.463 \mathrm{~cm}^{3}$, initial solution weight $M_{0}=0.439 \mathrm{~g}, S=15.2 \mathrm{~cm}^{2}$

TABLE 1. The characteristic size of the film nanostructure elements

\begin{tabular}{|c|c|c|c|}
\hline Nanoparticle type & $M_{C} / M_{0}$ & $l_{k o}, \mathrm{~nm}$ & $l_{k M}, \mathrm{~nm}$ \\
\hline nano & 0 & $21 \pm 2$ & $45 \pm 1$ \\
\hline micro & 0 & $280 \pm 20$ & $750 \pm 20$ \\
\hline nano & $3 \cdot 10^{-4}$ & $24 \pm 2$ & $53 \pm 2$ \\
\hline nano & $1 \cdot 10^{-3}$ & $26 \pm 2$ & $65 \pm 2$ \\
\hline
\end{tabular}

TABLE 2. The parameters of the differential distribution functions of nanoparticles microrelief on size

\begin{tabular}{|c|c|c|c|c|}
\hline Body type & $l_{k o}, \mathrm{~nm}$ & $A_{k}, \mathrm{~nm}$ & $p_{k}, \mathrm{~nm}$ & $n_{10}$ \\
\hline film nanoparticle & $21 \pm 2$ & $43 \pm 1$ & $2,0 \pm 0,2$ & $(3,1 \pm 0,1) \cdot 10^{8}$ \\
\hline film microparticle & $280 \pm 40$ & $690 \pm 10$ & $30 \pm 3$ & $(7,4 \pm 0,5) \cdot 10^{9}$ \\
\hline
\end{tabular}

where $l_{M 0}$ is the size in the absence of fullerene.

\section{Discussion}

The obtained data characterize the near-surface area of the system, which at the beginning of the process was the initial solution, and in the end, was an ordered layer of microparticles that are shown in Fig. 2. The change in the properties of the near-surface area occurred in two stages, as indicated by Fig. 3 and equation (13). According to equation (13), processes in the subsurface solution did not affect the flow $J$ of evaporated solvent molecules at $t<t_{1}$ and reduced the flow to the $J_{1}=0$ for $t>t_{1}$, and the function $J(t)$ underwent the change at $t=t_{1}$, similar to the changes that take place at the border in supersaturated 


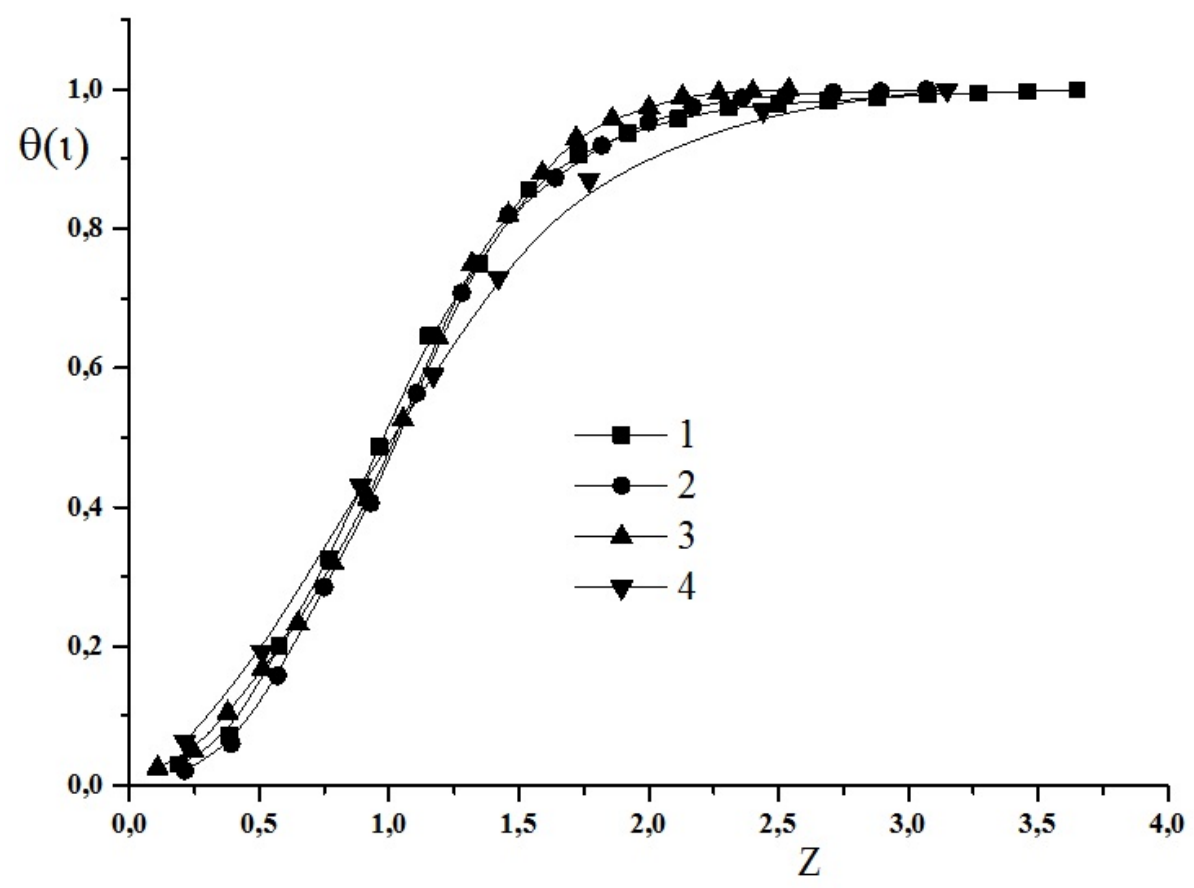

FIG. 4. Integral distribution functions of the structural elements of the films microrelief on size. 1 - nanoparticles; 2 - nanoparticles with fullerene content $3 \cdot 10^{-4} ; 3$ - nanoparticles with fullerene content $1 \cdot 10^{-3} ; 4$ - microparticles

metastable environments. Given these facts, we can assume that in the first stage $\left(t<t_{1}\right)$, the formation of the nanoparticles and their aggregation onto the microparticles took place, and in the second stage $\left(t>t_{1}\right)$, the enlargement of microparticles and their association in the aggregates dominated, forming the nanostructure which is shown in Fig. 2. This conclusion needs to be made to explain the proximity of the $\theta_{1}(l)$ functions of nanoparticles within the microparticles of different sizes (Figure 4). This closeness indicates that the microparticles were formed surrounded by nanoparticles which have acquired a stable $\theta_{1}(l)$ function prior to their significant association in the microparticles, i.e. in the first stage of the process. With this in mind, the data in Fig. 4 can be regarded as an appearance of the film's morphological memory, which preserves the information about the two-step process. The presence of morphological memory is also witnessed by formula (15), which allows one to determine the quantity of the fullerene in the system during film formation using the data on the $\theta_{1}(l)$ functions. Information on the kinetics of nano- and micro-particle formation can be extracted from formula (14). This formula is a solution of the equation:

$$
\frac{\partial \psi_{k}(l)}{\partial t}=D_{k l} \frac{\partial^{2} \psi_{k}(l)}{\partial l^{2}}-G_{k l} \frac{\partial \psi_{k}}{\partial l}
$$

at the boundary condition:

$$
\left[G_{k l} \psi_{k}(l)-D_{k l} \frac{\partial \psi_{k}(l)}{\partial l}\right]_{l \rightarrow l_{0 k}}=\delta(t)
$$

where $G_{k l}$ and $D_{k l}$ are the parameters satisfying the conditions $\int_{0}^{t} G_{k l} d t=A_{k}$ and $D_{k l} / G_{k l}=$ $p_{k} ; \delta(t)$ is the Dirac function. Equation (16) can be obtained using the relations (3)-(6), similar to the equation (7), assuming that $W_{j k}=0$, div $\vec{J}_{j n}=0, n=\pi l^{3} / 6 v_{A j}$, and

$$
G_{k l}=a_{k} \alpha_{k l}-b_{k} \beta_{k l}
$$




$$
D_{k l}=1 / 2\left(a_{k}^{2} \alpha_{k l}+b_{k}^{2} \beta_{k l}\right.
$$

Here, $a_{k}$ and $b_{k}$ are the effective changes of $l$ at the single acts of integration and disintegration of the particles; $\alpha_{k l}$ and $\beta_{k l}$ are the repetition frequency of these acts, independent on $l$, so that:

$$
p_{k}=\frac{a_{k}}{Z_{k}}\left(1+q_{k}^{2} Z_{k}\right) /\left(1-q_{k} Z_{k}\right)
$$

at $Z_{k}=\beta_{k l} / \alpha_{k l}$ and $q=b_{k} / a_{k}$.

These facts allow us to assume that the groups of nanoparticles in the first stage and the microparticles in the second stage were formed according to the same accretion mechanism (i.e. attaching of small particles to larger particles). This is indicated by the fact that the distribution of nanoparticles as well as microparticles is described by equation (14), characterizing a plurality of particles, each of them is coarsened by attaching the fragments significantly smaller than the particle itself. According to relations (14), (15) and (17), the nanoparticle nuclei were in the initial solution or were formed after the start of evaporation during the time much smaller than $t_{1}$. Their parameter, $n_{10}$, can be estimated, assuming that $n_{10}=\frac{\pi}{6} l_{10}^{3} / v_{A k}$, where $v_{A k}$ is the volume of the styrene molecule in its crystal. Doing so gives a figure of $n_{10}=(3.1-3.2) \cdot 10^{8}$ styrene molecules.

Based on equations (7), (14), (16)-(20), the nanoparticles on the first stage and the microparticles on the second stage undergo accretion independently $\left(W_{j k}=0\right)$ at homogenization of the near-surface solution areas at $t>t_{1}\left(\operatorname{div} \vec{J}_{j n}=0\right)$. This finding is consistent with formula (13). Additionally, relations (7), (14) and (16) indicate that the rate of integration of nano- and microparticles does not depend on their size. According to formulas (3)-(7), the independence of $D_{k l}$ and $G_{k l}$ from $l$ may be due to the fact that the nanoand microparticles had constant number of active centers, to which the macromolecules attached. As such centers, the nanoparticles could contain the ends of macromolecule chains, from which they consisted of, collected in the groups responsible for the independence of the parameters $a_{k}$ and $b_{k}$ on $l$ and for the formation of chains of nanoparticles, from which the microparticles were composed.

According to the model ((1)-(7)), the independence of $D_{k l}$ and $G_{k l}$ on $l$ does not exclude their dependence on evaporation time. This dependence is reflected by the parameters $A_{k}$ and $p_{k}$ in equation (14). Equation (14) is a solution of equation (16) for an arbitrary function $A_{k}(t)$ and a constant parameter $p_{k}$. According to the formulas (17)- $(20)$, the constancy of the parameter $p_{k}$ may be due to the fact that the frequency functions $\alpha_{k l}(l, t)$ and $\beta_{k l}(l, t)$ are such that the values $Z_{k}$ and $q_{k}$ are constant or $Z_{k} q_{k}<<1$. More detailed information on the frequency functions in the model $((3)-(20))$ cannot be extracted from the data on microrelief. However, it can be assumed that the function $\Psi_{1}(l)$ characterizes the primary aggregates of macromolecules $(j=2)$, as indicated by the value $n_{10}$, and the function $\Psi_{2}(l)$ refers to the ordered groups of aggregates (secondary aggregates $j=3$ ). Given this, one can proceed from the function $\Psi_{1}(l)$ to the distribution $\varphi_{2}(n, t)_{s}$, using the condition:

$$
\int_{l>l_{10}} \Psi_{1}(l) d l=\frac{1}{N_{2}} \int_{n>n_{10}} \varphi_{2}\left(n, t_{F}\right)_{s} d n,
$$

where $N_{2}$ is number of primary aggregates per unit volume of the near-surface layer of the film at $t=t_{F}$. A similar relation leads to a transition from the function $\Psi_{2}(l)$ to the distribution $\varphi_{3}(n, t)_{s}$. At the transition from $\varphi_{3}(n, t)_{s}$ to $\varphi_{3}(\vec{X}, \tau)_{s}$ it can be taken that $P_{3}\left(\vec{X}, t_{F}, \tau\right)=1$. 


\section{Conclusions}

It has been established that by evaporation of $o$-xylene and toluene solutions of polystyrene, polymeric bodies are formed which have a two-tier hierarchical nanostructure microrelief, and each level of the nanostructure has a stable distribution function of its structural elements on size. The results of these functions' determination coincide with the solution of the kinetic equations for the model of polymer body formation by evaporation of the polymer solution, beginning with the primary aggregates of macromolecules (nanoparticles), continued with the association of nanoparticles into the secondary aggregates of macromolecules (microparticles) and completed with the association of microparticles in the polymer bodies.

This model is based on the conservation of the number of atoms in the system and on the consolidation of nano- and microparticles as a result of binary collisions, and does not contain arbitrary assumptions. Therefore, the fit of the model to the experimental data can be considered as an indication of the fact that these processes actually took place during evaporation of the solution of polystyrene, and nano- and microparticles retained the information on how they were being consolidated, and this information can be obtained without destroying the film, defining the distribution function of the structural elements of the microrelief on size. Research methods used in this paper are not specific to the polystyrene. There are a wide range of polymers which have electron micrographs published in the literature that show microrelief, thus making them prime candidates for study using the above-described model.

\section{Acknowledgment}

The work was supported by Russia Foundation for Basic Research (grant N 15-4303034-a.

\section{References}

[1] Entelis S.G., Evreinov V.V., Kuzaev F.I. Reactive oligomers, M.: Chemistry, 1985, 304 p.

[2] Matyiaszewski K., Controlled/Living Radical Polymerization. Oxford University Press, Oxford, 2000, $416 \mathrm{p}$.

[3] Nalwa H.S. (Ed) Handbook of Nanostructured Materials and Nanotechnology. Organic, Polymer and Biological Compounds. Boston: Acad Press. 2000, 5, P. 577-583.

[4] Osada Y, Khokhlov A.R. (Eds) Polymer Gels and Network. Marcel Dekker, New York Basel, 2002, 309 p.

[5] Volynsky A.A., Bakeev N.F. Structural self-organization of amorphous polymers. Moscow, FIZMATLIT, 2005, 232 p.

[6] Caruso F.(Ed.), Colloids and Colloid Assemblies. Wiley-VCH, Weinheim, 2004, 603 p.

[7] Kabanov V.A., Selected Works. M.:Nauka, 2010, 432 p.

[8] Korolev G.V., Mogilevich M.M. Three-dimensional radical polymerization. Mesh and hyperbranched polymers. Chemistry: St. Petersburg. 2006, 334 p.

[9] Mezhikovsky S.M. Physical-chemical reactivity of oligomers. M.:Nauka, 1998, 233 p.

[10] Grosberg A.Y., Khokhlov A.R. Polymers and biopolymers from the point of view of physics. Intellect Publishing House, Dolgoprudny, 2010, 303 p.

[11] Zaitsev S.Yu., Zaitseva V.V. Supramolekulyrnye momomernye sistemy na osnove stirola I ikh komplksnoradikalnaya sopolimerixasiya (Styrene-Based Supramolecular Monomer-Polymer System and Their Complex-Radical (Copolymerization), Krasand, Moscow, 2012, 312 p.

[12] Acadsky A.A., Kondrashchenko V.I. Computational materials science of polymers. M: Nauchny mir, $1999,544 \mathrm{p}$. 
[13] Zherenkova L.V., Khalatur P.G., Khokhlov A.R. On the theory of polymer-containing dispersed systems. Aggregation of dispersed particles at the adsorption of macromolecules and the formation of ordered structures. Russ. Colloid Journal. 1997, 59(5), P. 634-645.

[14] Moor R.B, Martin C.R. Anal.Chem. 1986, 58, P. 2568-2580.

[15] Tobita H., Fundamental Molecular Weight Distribution of RAFT Polymers. Macromol. React. Eng. 2008, 2(5), P. 371-381.

[16] Liu C., Qin H., Mather P.T. Review of progress in shape-memory polymers. J. Mater. Chem. 2007, 17(16), P. 1543-1558.

[17] Xie T. Tunable polymer multi-shape memory effect. Nature, 2010, 464, P. 267-270.

[18] Jinlian Hu, Yong Zhu, Huahua Huang, Jing Lu. Recent advances in shape-memory polymers: Structure, mechanism, functionality, modeling and applications. Progress in Polymer Science, 2012, 37, P. 17201763.

[19] Melikhov I.V., Berliner L.B. Kinetics of periodic crystallization in the presence of crystals growing with fluctuating rates. Teor.Osn.Khim.Tekhnol. 1985, 19(2), P. 158-165.

[20] Melikhov I.V. Physico-chemical evolution of solid matter. M: Binom, 2006, 309 p.

[21] Alekseeva O.V., Bagrovskaya N.A., Kuz,min S.M., Noskov A.V., Melikhov I.V., Rudin V.N. The Influence of Fullerene Additives on the Structure of Polystyrene Films. Russ.J. Phys. Chem. A, 2009, 83(7), P. $1170-1175$.

[22] Kratschmer W., Huffman D.R. Production and Discovery of Fullerene: New Forms of Crystalline Carbon. Phil. Trans. Poy. Soc.(London) A, 1993, 343(1667), P.33-38.

[23] Melikhov I.V., Berliner B.L. Simulation of Batch Crystallization, Chem. Engineering Sci., 1981, 36, P. 1021-1024.

[24] Melikhov I.V. An evolutionary approach to the creation of nanostructures. Nanosystems: physics, chemistry, mathematics. 2010, 1(1), P.148-155. 